\title{
An insight into internet sector in Iraq
}

\author{
Ruaa Alsabah', Mustafa Aljshamee ${ }^{2}$, Ammar M. Abduljabbar ${ }^{3}$, Ali Al-Sabbagh ${ }^{4}$ \\ ${ }^{1}$ University of Kerbala, College of Computer Science and Information Technology, IT department, Kerbala, Iraq \\ ${ }^{2}$ Computer Science Department, Rostock University, Rostock, Germany \\ ${ }^{3,4}$ Ministry of Communication, Iraqi Telecommunications and Posts Company (ITPC), Baghdad/Babil, Iraq
}

\section{Article Info \\ Article history: \\ Received Jan 24, 2021 \\ Revised Apr 15, 2021 \\ Accepted May 10, 2021}

\section{Keywords:}

Internet gateway

Internet management

Internet service provider

Internet traffic

\begin{abstract}
The internet is considered to be the most advanced technology today and a gateway to modern communication and the sharing of information, products, services, and technology. Nowadays, users want to be able to access anywhere and anytime several services and applications, which is increasing data traffic and triggering a mobile data explosion. Iraq has major problems in increasing the growth and use of the internet and changing the standard method of communication. This is a big challenge, however, since there are several variables that characterize this phase of transformation. In this paper, the problems, vision, and solutions are presented in details. This study aims to clarify the factors of internet use in Iraq by the use of an acceptable approach and by suggesting new solutions for all the presented problems. This work also, clarify the expected traffic and the mechanism to transform the traffic between local ISP's networks (AS) internet exchange points.
\end{abstract}

This is an open access article under the CC BY-SA license.

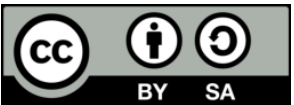

\section{Corresponding Author:}

Ali Al-Sabbagh

Ministry of communication

Iraqi Telecommunications and Posts Company (ITPC)

Babil, Iraq

Emil: aalsabbagh2014@my.fit.edu

\section{INTRODUCTION}

Nowadays the internet technologies are very essential in human life because all the digital technologies activities depend on it such as watching YouTube, online shopping, connecting to bank accounts, video calling, Facebook, Google's services, and others [1], [2]. Furthermore, there is a huge impact of information and Communication on societies' economics. For the previous reason, the internet's technologies subscribers all around the world request and strongly need a better internet by providing better reliability and high bandwidth [3], [4]. In Iraq, the story of the internet is complicated since the progress of it was straggled with many wars in addition to the stranglehold of state censorship.

Before 2003, the internet was very limited; however, because of infrastructure and technical problems, the internet still not available to everybody. On the other hand, since the internet is affected by the unstable conditions for Iraq, this makes it hard to be a full potential of widespread. For this reason, a slow step is being made to create a future-looking technological foundation [5], [6]. After the war 2003, Iraq shows clear upgrade in a short period, represented by the transformation from an isolated society and technologically backward into a globally connected nation with the accepted potential to grow. This is all because access to the internet has begun and made a huge difference in many fields. To increase society internet usage, Iraq developed different procedures to get to best benefit from the advancement in the global communication system [1], [2], and [7]. Although Iraq continues suffering from economic and political challenges until this time especially in some liberated areas, civil stability during 2020 might give the chance 
to restore, rebuild and fix sectors for the damaged telecommunications services where networks had been destroyed.

The late process of writing Iraq's Telecommunications Laws made the situation worst since it has been under scrutiny for many years and has not finished yet. In early 2020; the law had been discussed at a workshop hosted by the GSMA, a suggestion of improving the law raised to improve and reach the best global practices. The neglected backbone infrastructure in Iraq causes a limitation in the internet growth, in contrast, there is an increased number in the wired, and data subscription. Because of the poor infrastructure, high cost of bandwidth and few options to choose from all these reasons leads to a low internet-subscription ratio [8]-[10].

Same as in the other country's internet in Iraq, play a major role in life, it is not only a basic thing, it is considered as a whole life of some people no matter who is using it and how it represents a different value for each one. For this reason, Iraq companies started to make many changes for internet development. Although many of them started to implement projects to enhance the internet infrastructure, the government must take some responsibility to support such a development. The problem of providing the internet is not only because of the poor infrastructure, but also a matter of interest, knowledge, and needs. This paper presents a technical explanation of the current MoC's (infrastructure of internet, limitations, problems, solutions, and future plans) on the internet in Iraq. These solutions and future plans could be a good beginning and bring good changes so the next years face big differences and widespread of the internet in Iraq.

\section{INTERNET CHALLENGES}

Currently, users want to be able to access anywhere and anytime several services and applications, which is increasing data traffic and triggering a mobile data explosion [1]. The statistics in Iraq show that internet usage is skipped rapidly to 19 million users in 2017, instead of 2.2 million users in 2012 . The current expected usage is more than 30 million internet users in 2020 and 41 million as cellphone connections [4], [6]. This section can be divided in two parts as the current status and future solutions:

\subsection{Current infrastructure and problems}

Iraq is one of the least countries in internet speed. The current total traffic is about 850-1000 Gbps through Ministry networks (fully controlled by Alsalam state company). This traffic comes via 13 border points (8 in Iraqi borders plus 5 in Kurdistan region). This state of internet development in Iraq is in a disappointing situation and needs rapid developments. There are two main reasons for this lack of services: First, most internet service providers (ISP) rely on free licensed wireless services as a last mile-access. Second, MoC has the right to license the optical fiber (monopoly) with very high prices compared to Iraqi neighbors [9], [10]. As a result, this will increase the cost of the internet for public usage. As mentioned in the latest reports of the Speed-test Global Index, which is compares internet speed data monthly around the world. The global statistics showed a maximum internet bandwidth this year of 197-150 Mbps for advanced countries like Singapore in 1st then Korea, Romania, Thailand, and Switzerland in 5th for fixed broadband. And for mobile internet 83-73 Mbps for United emirates $1^{\text {st }}$ rank, South Korea, Qatar, China, and Canada in $5^{\text {th }}$ rank.

In November, Iraq ranked as $105(-4)$ in fixed broadband and 133 (-6) in mobile internet speed services. Since November 2019, the speed variation in mobile and fixed internet access is illustrated in Figure 1. In November 2020, the monthly average in mobile services is 11.98 and $5.71 \mathrm{Mbps}$ (with $52 \mathrm{~ms}$ latency) for download (green line) and upload (purple), respectively [11], [12]. The figure also shows in fixed broadband service is 27.85 and $25.65 \mathrm{Mbps}$ (with $23 \mathrm{~ms}$ latency) in download and upload, respectively [13], [14].

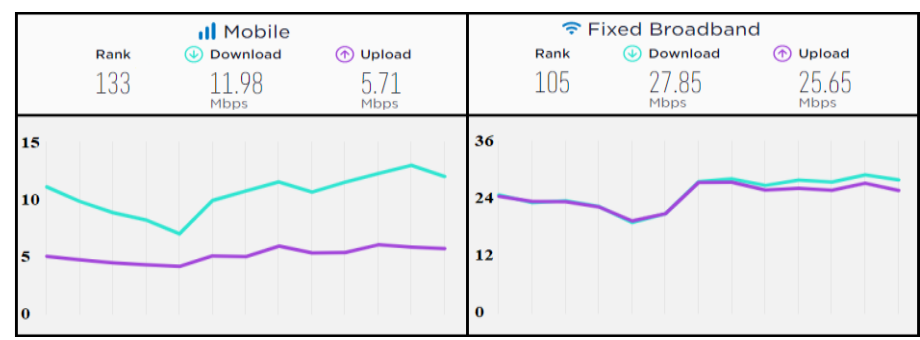

Figure 1. Internet speed variation (mobile and fixed broadband) [12] 
As mentioned earlier, the internet service comes from several ISP companies through MoC Infrastructure. Then, ISP provides the internet to multi regions by resellers with multi Wi-Fi towers. Currently, Iraq has around 8000 resellers that providing the service by more than 20000 registered towers. Map distribution is presented in Figure 2. Besides that, there are several towers that are not registered with the Iraqi government. These towers cover around a half kilometer causing severe interference.

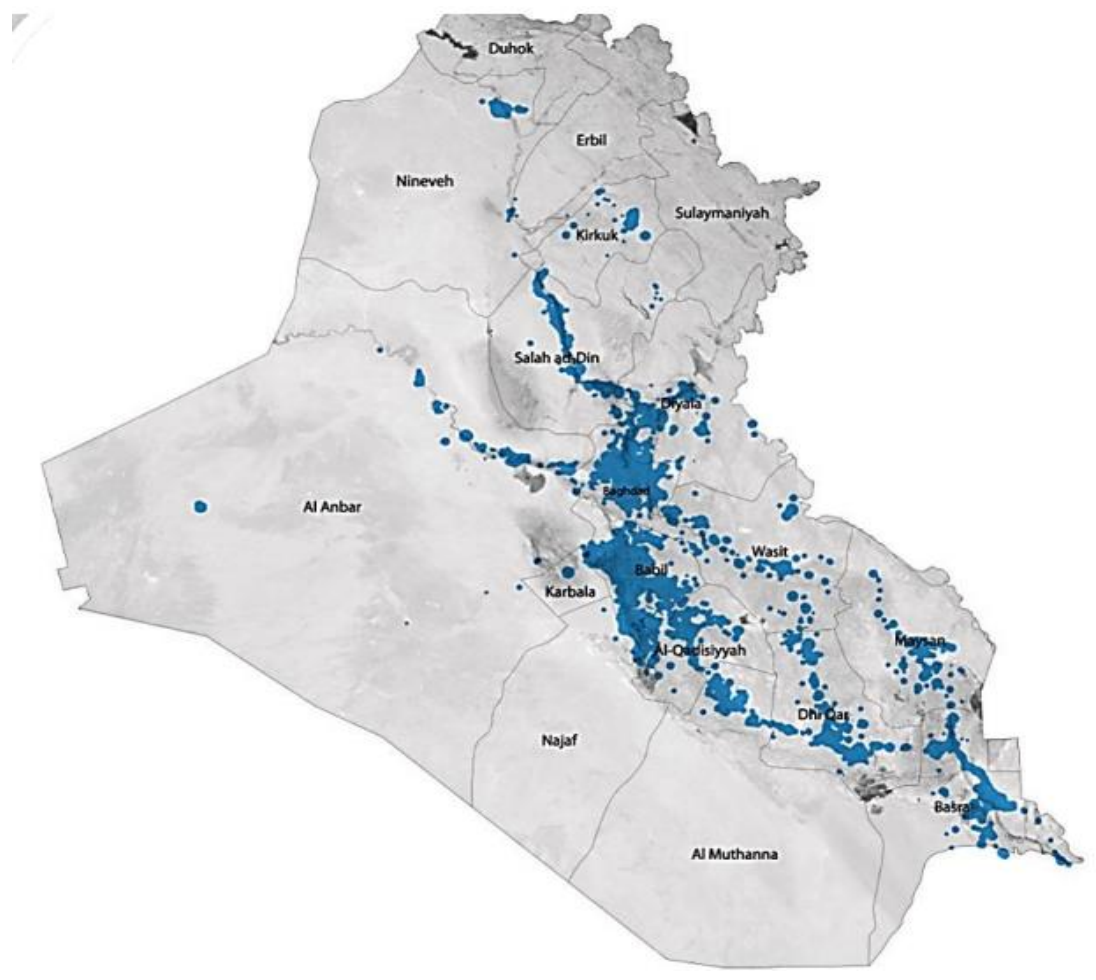

Figure 2. Map distribution of internet resellers [source: MoC]

From the technical side, as known Wi-Fi technology uses radio waves to connect devices. And two significant parameters in the internet wireless service, which are the bandwidth and range. The range; defines the maximum coverage area, whereas more bandwidth is required for higher download and upload over the wireless network.

Two different frequencies are used in Iraq: $2.4 \mathrm{GHz}$ and $5 \mathrm{GHz}$. In $2.4 \mathrm{GHz}$, there are only three non-overlapping channels possible that can be seen; also, more devices such as microwaves and cordless phones are using the same frequency. This will lead to limited bandwidth and very high interference, but with low cost, since it is cheaper to produce such devices, and larger coverage, because the radio waves can penetrate walls and floors with respect to the $5 \mathrm{GHz}$. The $5.8 \mathrm{GHz}$ is used recently after the interference and low bandwidth issues. It has a higher bandwidth with more channel available (about 23). Also, this frequency is not used by common wireless devices like the $2.4 \mathrm{GHz}$; therefore, there will be a little interference to cause a reduction in bandwidth, i.e. it can carry more data and transmits with faster speed. The main disadvantage of this frequency is the low coverage area [15], [16].

\subsection{Technical solutions}

The technical solutions of low-speed internet in Iraq are summarized in two directions. The first solution is to use licensed frequencies instead of free Wi-Fi channels. This is known as fixed wireless access (FWA), which provides a broadband wireless access (BWA) using specific frequencies that enable higher data rate, wide range, low interference, higher capacity, and can be utilized by both public and governmental sectors. The license for this solution can be granted and organized by the communication and media commission. The second solution is to use wired solutions such as fiber-to-the-home (FTTH), fiber-to-thecabinet (FTTC), or very high data rate digital subscriber loop (VDSL). Recently, MoC started some regional FTTH projects that located in Baghdad and other provinces with four available profiles of speed 3.5-10 Mbps. Figure 3 presents and described the scope of the project. 


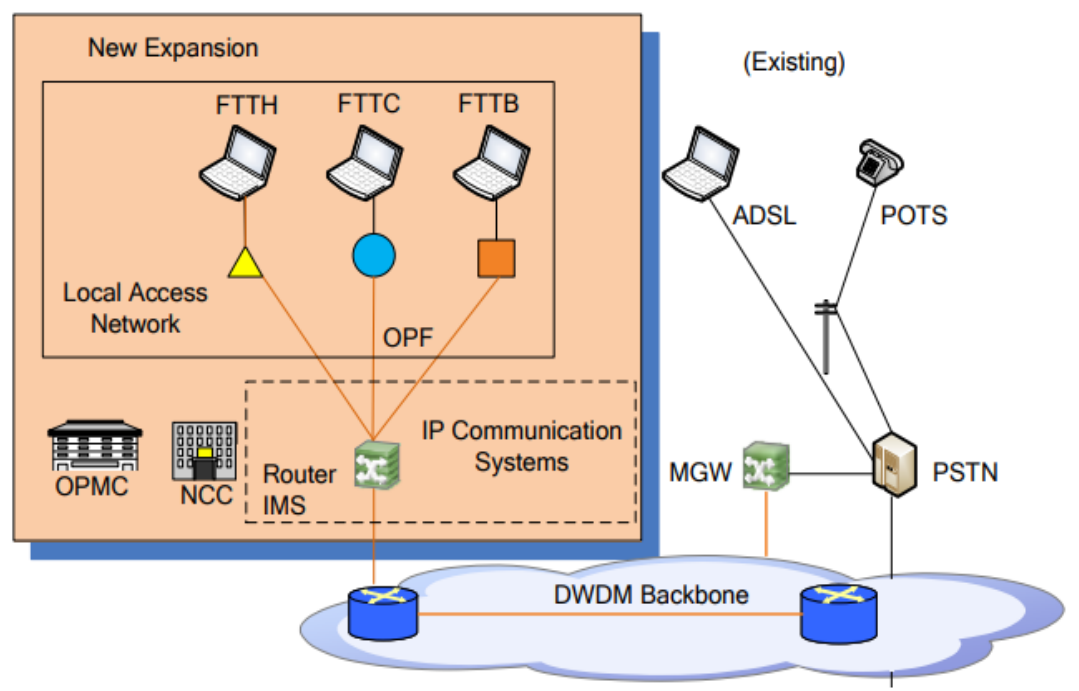

Figure 3. Scope of current FTTH project [source: MoC]

Local access network local access network is composed of FTTC (cabinet), FTTB (building), and FTTH (home) that can supply a totally $286 \mathrm{~K}$ lines. Also, the abbreviations in the figure can be described as follow: New communication center (NCC), outside plant maintenance center (OPMC), dense wavelength division multiplexing (DWDM), public switched telephone network (PSTN), asynchronous digital subscriber line (ADSL), plain old telephone service (POTS), IP multimedia subsystem (IMS), and media gateway (IMG).

The main problem with these contracts is the duration of investment, which is five years only, and it should be at least 10 years and above. The FTTH/C can present a suitable methodology, at least for the midlevel planning. This technology can provide a very secure and reliable service. In future, such services can offer a $20 \mathrm{~km}$ reach up to (2.25-2.5) Gbps as a download/upload, with gigabit passive optical networks (GPON) optical access network and multi-services such as internet, TV, and telephone. Meanwhile, FWA is more cost-efficient as compared with the latest mentioned wired access techniques with some limitations. The wired connection will help to decrease the number of wireless subscribers. The current growth of the optical fiber networks is very slow because of two main reasons: First, the monopoly of MoC in very high prices to Iraqi neighbors, MoC should more liberate the fiber optics to the private sectors with secured regulations to get better internet services. Second, installing underground cables is the only allowable legal way [17].

\section{FINDINGS}

In 2014 Iraq has established (very lately), autonomous system number (ASN), to control the internet streaming for all ISP companies. ASN is a unique number, a collection of connected internet protocol routing under the control of network operators on behalf of a single administrative unit. The nodes inside any autonomous system communicate through an internal gateway protocol (IGP). The AS is assigned a globally unique identification number by the internet assigned numbers authority (IANA) through one of five global regional internet registries (RIRs) set up for Africa, America, Asia, Latin America and the Caribbean, and Europe [18], [19]. Until 2007, ASN was 16 bit (2 byte) and gave IANA 65,536 possible ASNs to distribute (IPv4 addresses). Once, IPv6 has been created, so the ASN became 32 bit (4 byte). This provides 4,294,967,296 autonomous system numbers. There are three types of Autonomous Systems: i) AS connects to two or more ASes, ii) AS connects to only one other AS (not visible to the rest of the internet), iii) A transit AS acts as a link between two or more other ASes, allowing for data to pass from even unassociated networks [17], [19].

Figure 4, this info-graph illustrates an example of traffic streaming via MoC's ASN, which is (AS208293) as peering with IPv4 and IPv6. Majority of IPv4 streaming is 18.9\% via Al-Sard Fiber services (AS39216), and then Nawroz Telecomm (AS21277) by $13.9 \%$ and the third is $7.9 \%$ by IQ Networks (AS44217). Also, the majority peering of IPv6 is $26 \%$ via Earthlink Telecomm (AS60051), then $16 \%$ by IQ Networks Ltd, and the third and fourth is $13 \%$ by Cogent Communication company (AS174) and Level 3 parent (AS3356) [20], [21]. 


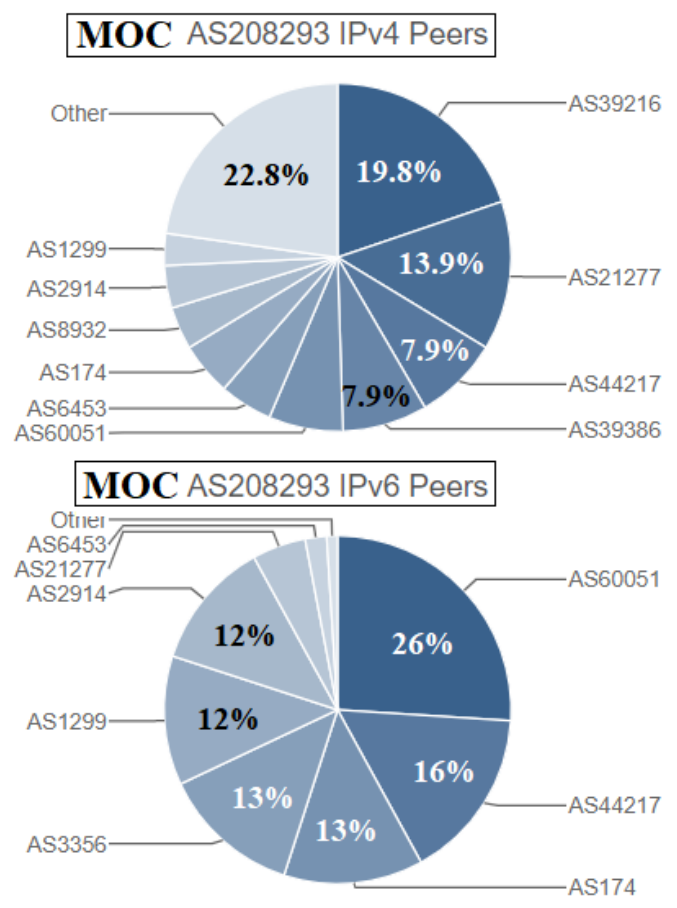

Figure 4. MoC ASN's percentage with ISP (IPv4 and IPv6) [21]

\section{RESULTS AND ANALYSIS}

This presented as the first step to initialize the international gateway in Iraq. As discussed below, the internet gateway, whether it has a distributed or central topology, requires to have some functionalities that can be achieved in the current gateway with only one layer. MoC should uses at very early stage, the border gateway protocol (BGP), which uses the ASN to uniquely identify each system. The BGP manages the routing of packets between different autonomous systems paths across the Internet.

It is a path vector protocol, and enabled AS in border routes send signals and from the external internet through a border gateway protocol (BGP) via IGP. Countries need to be protected from distributed denial of service (DDoS) attacks by using a robust Anti-DDoS system. DDoS attacks could be either locally or coming from the internet. In the case of large attacks, a cloud-based Anti-DDoS system could be used for mitigation. Moreover, the security solution for the international internet gateway (IIG) must include data packet inspection (DPI) system. DPI gives the ability to filter websites based on content and services (e.g. Voice over IP) which optimizes the traffic eventually. Finally, it is worth to mention that a logging system could be used for security and traffic optimization as well by analyzing, studying and tracing the logs in order to create future upgrades and enhancements. Iraq should increase and optimize the performance of internet across the country by several steps including but not limited to:

- Controlling the full bandwidth of internet traffic coming into Iraq.

- $\quad$ Protecting from cyber-attacks and eliminating fraud using security services such as Anti-DDoS, DPI, and logging system.

- $\quad$ Offering a secure backbone infrastructure for ISPs and government sector.

- Providing a secure digital infrastructure for e-commerce.

- $\quad$ Expecting a 5 Tbps internet traffic in the near future and upgrade the infrastructure.

- $\quad$ Building internet exchange points (IXPs) to transport Internet traffic between local ISPs networks (AS).

One of the benefits of using IXP is when one of the ISPs hosts a website for government/private sectors; other ISPs can access the website locally without the need to request it from outside the country. This optimizes the internet traffic going in and out the country. However, ISPs have to be able to exchange traffic between themselves through a local IXP [14].

In summary, international gateways allow connections to international cable systems (often satellite or submarine) to organize the voice and data traffic. Iraq and most developed countries are connected by several redundant international links, which are often available at internet exchange points to receive internet traffic to MoC's infrastructure. National networks then connect between IG and access networks to provide internet services. Figure 5 illustrated as the function of IIG. 


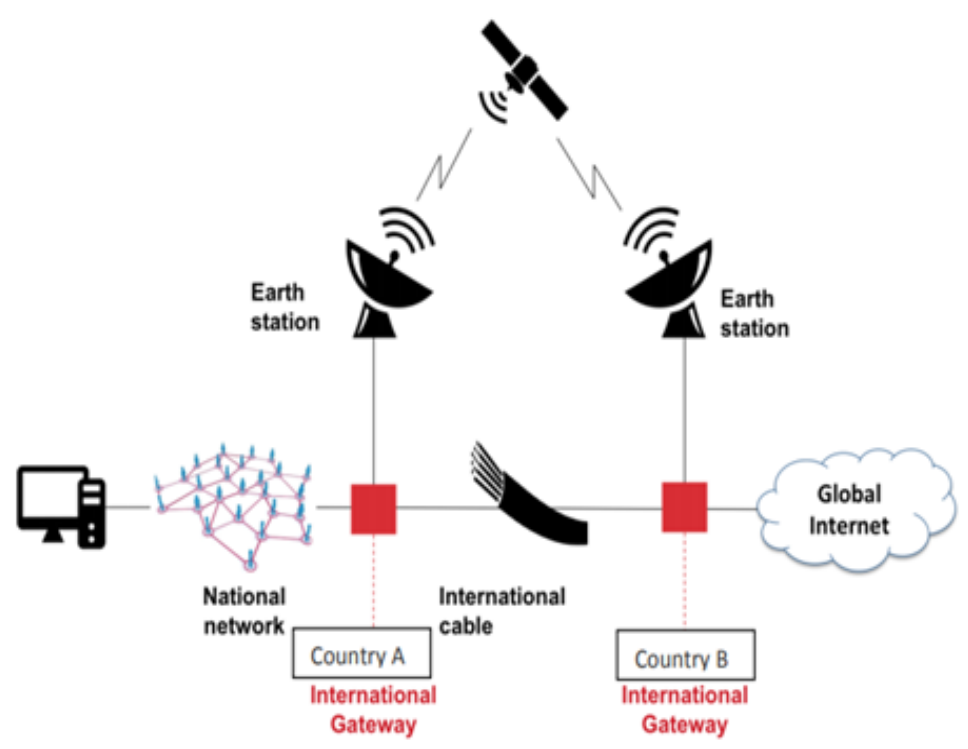

Figure 5. Function of an international gateway [22]

These points are achieved by building a robust IIG with modern security equipment and software. However, multiple protocols and different devices brands make the networks complicated. Therefore, a layered model for a reliable network was designed and developed by [23], [24] to maintain a scalable and cost-effective network. This model shown in Figure 6 has three layers including:

- Access layer (Aggregation) incorporates all ISPs networks to be connected to this layer. The ISPs traffic will either be redirected nationally or internationally.

- Distribution layer serves as the communication point between the core and the access layer. Its key functions are to provide routing, filtering, and WAN access to determine how packets can reach the core.

- The DPI and Anti-DDOS systems need to be located between the internet layer and the distribution layer to inspect the traffic before forwarding it to its destination.

- Core layer (internet) referred to as the network backbone; which is responsible for transporting large amounts of traffic quickly and to be connected to our national DWDM [24], [25].

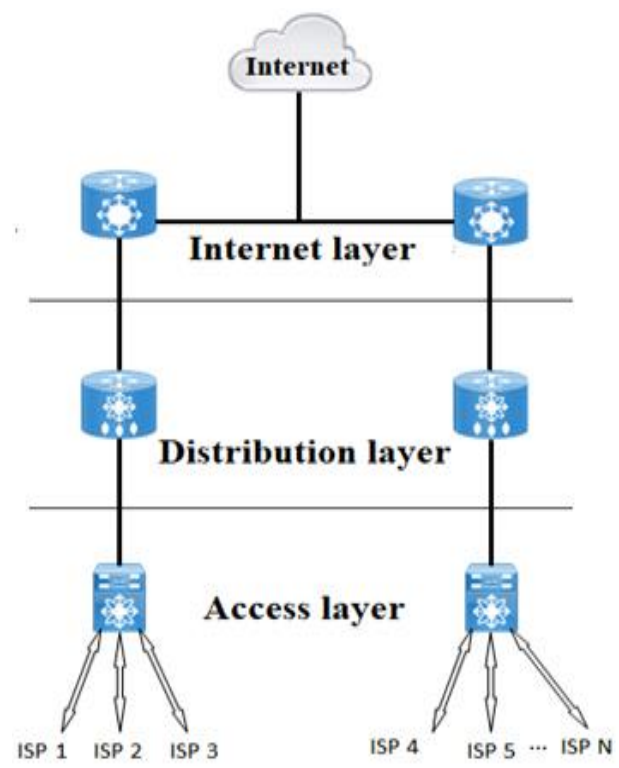

Figure 6. Cisco three layers model 


\section{CONCLUSION}

The performance of the internet in Iraq should be increased and optimize all around the country by several steps such as; the full bandwidth must be controlled for the internet traffic that is coming to it, use a security service to eliminate fraud and preventing from cyber-attacks, secure the infrastructure of the backbone for the government sector and ISPs, the e-commerce, and others must have a secure digital infrastructure, the internet traffic expected to be a 5 Tbps so the infrastructure should be upgraded, to transport internet traffic between local ISP's networks (AS) internet exchange points (IXPs) must build.

\section{REFERENCES}

[1] H. Al Bayati and G. Felgenhauer, "Iraq Business Perspectives (Part 1): Internet Infrastructure and Stock Market Modernisation," Iraq energy institute (IEI), Jun. 2019.

[2] R. Lee, S. Fox, and D. Fallows, "The Internet and the Iraq war," Washington, DC: Pew Internet and American Life Project, pp. 1-11, 2003.

[3] F. H. Al-Hammadany and A. Hesmhmati, "Analysis of the purpose of using Internet in Iraq: A multinomial logit model," Journal of Knowledge Management, Economics and Information Technology, vol. 1, no. 6 pp. 1-41, 2011.

[4] F. H. Al-Hammadany and A. Heshmati, "Determinants of Internet use in Iraq," International Journal of Communication, vol. 5, 2011, Art. no. 23.

[5] "Internet Usage and Marketing Report," Internet World Stats. Accessed: Nov. 25, 2020 [Online]. Available: https://www.internetworldstats.com/me/iq.htm.

[6] Simon Kemp, "DIGITAL 2020: IRAQ," Data Reportal. Accessed: Dec. 15, 2020 [Online]. Available: https://datareportal.com/reports/digital-2020-iraq.

[7] B. Rashid, A. Faraj, and W. Shareef, "Investigating and Evaluating Internet Usage in Kurdistan Region of Iraq," International Journal of Multidisciplinary and Current Research, vol. 4, pp. 474-479, 2016.

[8] B. A. Tawfeeq, M. Q. Kheder, and N. N. Qader, "Internet governance from the regional kurdistan of Iraq," Int. J. of Multidisciplinary and Current research, 2014.

[9] C. Avgerou, "The link between ICT and economic growth in the discourse of development," in Organizational information systems in the context of globalization, Springer, Boston, MA, 2003, pp. 373-386, doi: 10.1007/978-0387-35695-2_23.

[10] M. Taie and S. Kadry, "E-Government: Latest Trend and Future Perspective The Iraq Case," European Journal of Scientific Research, vol. 99, no. 2, 2013, Art. no. 307.

[11] S. Rashid, "An overview on internet in Iraq," HCU| Hamburg, REAP Masters, WS, The World Online, 2012.

[12] "Speedtest Global Index", Speedtest. Accessed: Dec. 15, 2020 [Online]. Available: https://www.speedtest.net/global-index/iraq\#fixed.

[13] N. Gelvanovska, M. Rogy, and C. M. Rossotto, "Broadband networks in the Middle East and North Africa: Accelerating high-speed internet access," The World Bank, 2014, https://www.worldbank.org/en/region/mena/publication/broadband-networks-in-mna. (accessed Jan. 01, 2021).

[14] W. Obile, "Ericsson mobility report.," Ericsson, 2016. Accessed Dec. 10, 2020. [Online]. Available: https://www.ericsson.com/en/mobility-report.).

[15] H. M. T. Al-hilfi, S. k. J. Al hummadi and M. S. Mustafa, "Effect of Modulation Techniques on the Performance of Voice and Video Service for WiMAX Networks in Baghdad," 2018 International Conference on Wireless Communications, Signal Processing and Networking (WiSPNET), 2018, pp. 1-3, doi: 10.1109/WiSPNET.2018.8538693.

[16] H. Alyasiri and N. Abdulbaqi, "Proposed policy for Iraqi ICT sector (part I)," 2012 International Conference on Future Communication Networks, 2012, pp. 129-134, doi: 10.1109/ICFCN.2012.6206855.

[17] Aurther D. Little, ”Workshop," [Online]. Available: https://www.adlittle.com/.

[18] "Internet-Assigned-Numbers-Authority (IANA)." Accessed Dec. 10, 2020. [Online]. Available: https://www.iana.org/about.

[19] M. Cotton, L. Eggert, J. Touch, M. Westerlund, and S. Cheshire, "Internet Assigned Numbers Authority (IANA) Procedures for the Management of the Service Name and Transport Protocol Port Number Registry," RFC, pp. 1-33, 2011.

[20] G. Huston, "Exploring autonomous system numbers," The Internet Protocol Journal, vol. 9, no. 1, pp. 2-23, 2006.

[21] "Hurricane Electric's internet services (International backbone)." Accessed Dec. 15, 2020. [Online]. Available: http://he.net/.

[22] "Effect of Open International Gateways on the Broadband Connectivity Market," Report of United Nations Economic and Social Commission for Asia and the Pacific (ESCAP), Bangkok, Thailand, Feb, 2017.

[23] S. Malisuwan, D. Milindavanij, and N. Tiamnara, "Thailand's International Internet Gateway (IIG): Market and Development." International Journal of Advanced Research in Management (IJARM), vol. 7, no. 1, pp. 24-33, 2016.

[24] "Campus Network for High Availability Design Guide," Design, Cisco Validated, 2008.

[25] N. Cascarano, L. Ciminiera, and F. Risso, "Optimizing deep packet inspection for high-speed traffic analysis," Journal of Network and Systems Management, vol. 19, no. 1, pp. 7-31, 2011, doi: 10.1007/s10922-010-9181-x. 